Les articles ci-après, rédigés par les médecins de famille Franz Marty et Bernhard Sorg, ont une approche critique du programme de formation continue au titre de médecin spécialisé en médecine interne générale; en effet, pour eux, ce programme est insuffisant du point de vue de la garantie de la relève des médecins de famille et de celui des compétences en médecine de famille. Afin d'élargir le débat sur ces questions, la rédaction a invité la Société Suisse de Médecine Interne Générale (SSMIG), société de discipline médicale compétente dans ce domaine, à prendre position, ainsi que d'autres experts. Vous trouverez ces articles dans les pages suivantes, sous la rubrique «Discussion».

La rédaction

\title{
Ce que nous enseigne la pratique
}

\section{Franz Marty}

Dr méd., membre de la FMH

\section{Introduction}

Les soins médicaux ambulatoires en Suisse ont été profondément métamorphosés durant ces 15 dernières années! Les changements structurels intervenus dans le paysage des cabinets [1] et l'ancrage aux institutions académiques ont ouvert de nouveaux débouchés professionnels et de nouvelles carrières académiques aux jeunes médecins de famille. La médecine de premier recours est aujourd'hui la discipline qui permet les possibilités professionnelles les plus variées, tout en offrant des modèles de travail très flexibles [2]! Malgré ces conditions de nature à attirer les candidats, le problème de la succession s'est fortement aggravé ces dernières années. Pourquoi cette filière est-elle délaissée des médecins? Le nouveau programme de formation postgraduée (PFP) en médecine interne générale (MIG) est-il trop porté vers le «secteur hospitalier»? Ou la formation de médecin de famille manque-t-elle d'attraits?

\section{La formation postgraduée en médecine de famille}

Dans la plupart des pays dotés d'un système de santé moderne, un titre en médecine de famille (MF) s'obtient à l'issue d'une formation postgraduée (FP) structurée de 5(-6) ans, répartis d'ordinaire en 2 ans de formation clinique de base et 3 ans de formation spécifique [3]. Outre ces médecins de famille titulaires d'une FP spécifique, la médecine de premier recours est exercée par les généralistes (FP min. de 3 ans) et, surtout dans l'espace germanophone, par des internistes généralistes.

\section{Le PFP en médecine interne générale}

En Suisse, la FP en médecine de famille, d'une durée de 5 ans, est régie depuis 2011 par le PFP en MIG. Elle consiste en une FP de base obligatoire de 3 ans en médecine interne générale suivie d'une FP structurée de 2 ans selon les préférences individuelles. La FP de base comprend 2 ans de MIG dans un hôpital (dont 1 an dans une clinique A) et au moins 6 mois de FP en ambulatoire (dans une polyclinique ou en assistanat dans un cabinet).

Une FP spécifique en médecine de famille est cependant facultative, et l'acquisition par le médecin de connaissances et d'aptitudes dans sa pratique ultérieure, une FP en chirurgie, y compris urgentiste, ainsi que l'extension des compétences cliniques par des rotations rapides dans différentes disciplines sont recommandées sans être obligatoires [4].

\section{Chiffres et expériences de 2011 à 2016}

\section{Nombre de nouveaux spécialistes en MIG}

Le nombre total de spécialistes en MIG [5] a augmenté, passant de 5800 à 5921 (+2\%) dans le secteur ambulatoire et de 1603 à 2295 (+43\%, tab. 1) en stationnaire. Leur nombre a baissé de $336(-6 \%)$ dans le secteur ambulatoire et augmenté de 685 (+43\%) dans le secteur hospitalier chez les moins de 65 ans, tandis qu'on note des hausses respectives de 457 (+44\%) et de $7(+16 \%)$ chez les médecins à l'âge de la retraite.

\section{Compétence des nouveaux spécialistes en MIG}

Une analyse des centres de FP choisis par les nouveaux spécialistes en MIG montre que seul un spécialiste sur dix, soit moins de trente par an, ont suivi les recommandations lors de leur formation. Ceux qui ont suivi une FP chirurgicale ne sont plus que 39\% (anc. 55\%), tandis que la part de ceux optant pour l'assistanat en cabinets est maintenue entre 30 et $40 \%$ [6]. 


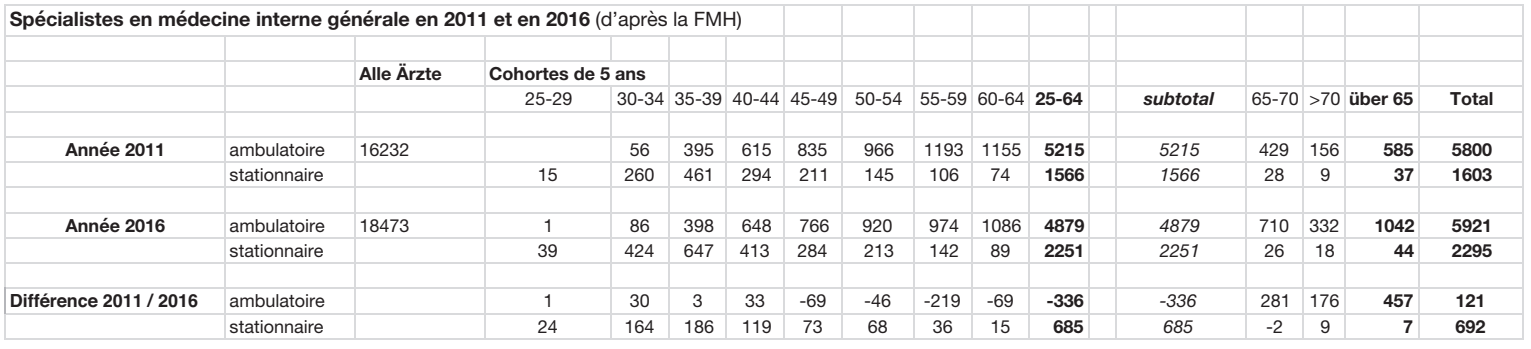

Tableau 1

\section{Nos expériences de l'assistanat en cabinet}

La demande de places d'assistanat a été très variable. Après un surplus de demandes de 2010 à mi-2013 suivi d'une période creuse jusqu'à début 2016, nous connaissons un équilibre approximatif entre l'offre et la demande depuis le printemps 2016.

Beaucoup d'assistants venus de petits hôpitaux ont fait leur demande d'assistanat dès la première moitié de leur temps de formation avec un objectif professionnel clair, celui de devenir médecin de famille. La plupart possédait un vaste savoir clinique et pratique. Les assistants venus de grands hôpitaux ont souvent fait leur demande d'assistanat sur le tard avant de constater leurs insuffisances (compétences en chirurgie et en clinique pour la plupart) et de réaliser qu'il serait difficile de les combler. Beaucoup sont restés ambivalents dans leur choix de carrière et ont opté ensuite pour un poste de chef de clinique interniste ou une activité ambulatoire dans une autre spécialité.

Les perspectives de carrière d'un groupe important de futurs médecins - les femmes ayant des enfants en bas âge - ont toujours été difficiles. Beaucoup possèdent une bonne formation interdisciplinaire mais sont empêchées de se spécialiser en MIG faute de postes à temps partiel dans les cliniques A.

On est frappé par la persistance d'une image d'infériorité de la carrière de médecin de famille, une situation très bien décrite par une jeune spécialiste en MIG dans une interview radiophonique sur SRF [7]:

"[Nous autres médecins de famille] devrions cesser de faire profil bas. Nous avons une mission très importante à remplir auprès du système de santé et de nos patients. Et notre problème d'image est d'avoir toujours le sentiment de n'être qu'un petit médecin de famille et que la vraie médecine est celle des spécialistes. Nous devons nous départir de cette image.»

\section{Conclusion}

Le nouveau PFP en MIG prive le secteur ambulatoire de médecins et restreint l'étendue des compétences que devrait conférer cette formation. Devenir un médecin de famille compétent demande plus d'efforts que la seule FP d'interniste généraliste. Or le titre de spécialiste en MIG atteste une telle compétence même chez les médecins qui ne l'ont pas acquise, d'où un profil professionnel mal défini vis-à-vis de l'extérieur et des problèmes d'identité professionnelle à l'interne.

Dans un tel contexte, les jeunes médecins n'ont que peu d'intérêt pour une FP adéquate en médecine de premier recours et une carrière de médecin de famille. L'image de la profession en souffre, la médecine de famille est le débouché de dernier recours!

\section{Que faut-il faire?}

Les facteurs décisifs pour rendre la FP attrayante sont: a) l'accès simplifié à une FP adéquate, b) un profil professionnel clair, c) la caractérisation des compétences acquises. Une formation au profil clair apportera une amélioration rapide et durable de l'image de la profession.

\section{Finaliser un PFP commun}

La manière la plus simple de réaliser un tel projet est de finaliser une forme commune du PFP de médecine interne générale telle qu'elle était prévue à l'origine. Il suffirait de compléter le PFP actuel d'un chapitre intitulé «FP obligatoire pour la carrière de médecin de famille». a) une FP de base sur 3 ans dont 2 ans de MIG, 6 mois dans un service d'urgence interdisciplinaire (MIG et chirurgie) et 6 mois d'assistanat en cabinet et b) une FP modulaire sur 2 ans dont (nouvelle exigence) au moins 6 mois dans une discipline supplémentaire. Tous les médecins qui achèvent la FP comme exigée actuellement obtiennent le titre de "spécialiste en MIG», et ceux qui accompliraient en plus la FP obligatoire pour médecins de famille obtiendraient le titre de «spécialiste en MIG et médecine de famille».

\section{Avantages d'une telle réglementation}

Une telle réglementation confère au spécialiste en médecine de famille une formation structurée spécifique au niveau individuel, un profil professionnel clair et donc une identité professionnelle, récompense son 


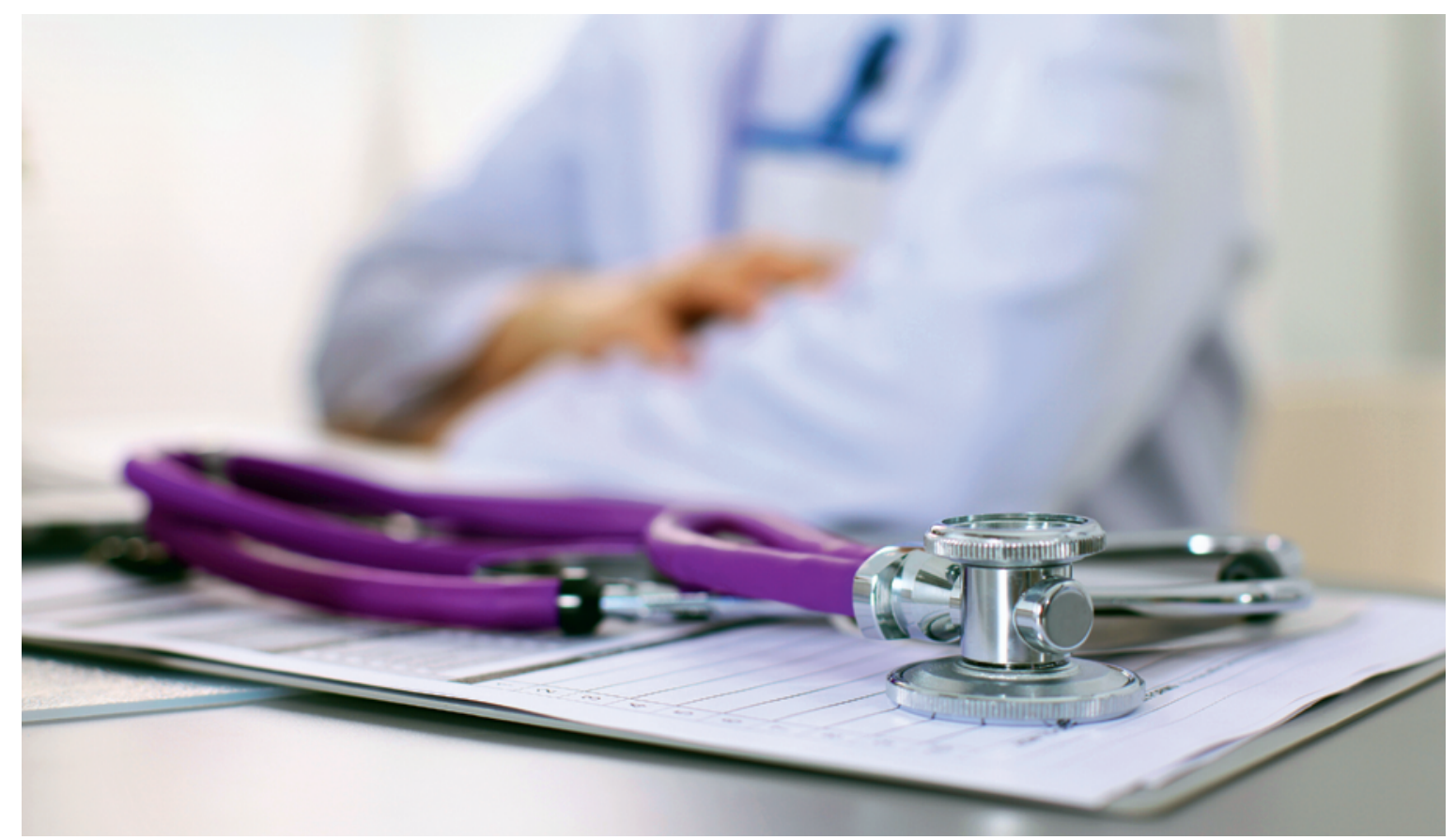

Comment rendre la formation en médecine de famille attrayante pour les jeunes médecins?

effort supplémentaire en lui donnant un statut défini, rend ses compétences médicales visibles de l'extérieur et facilite une FP répondant aux objectifs. Au niveau institutionnel, elle améliore la coordination entre les secteurs stationnaire et ambulatoire, par ex. au moyen d'incitatifs pour des offres de FP combinées (parex. une année de formation A offerte par une clinique A et suivie d'un assistanat en cabinet). Elle encourage l'interdisciplinarité et l'interprofessionnalité en médecine et permet de s'assurer les compétences des médecins de famille pour administrer les soins de santé de premier recours.

En procédant ainsi, on évite aussi et surtout de se créer de nouveaux problèmes: des processus existants (examens, classification des sites de FP, etc.) peuvent être repris sans modifier la structure de la FP et tout en préservant la compatibilité avec l'UE. La "perméabilité» professionnelle reste acquise: tous les spécialistes en MIG peuvent continuer de pratiquer des soins ambulatoires de premier recours et tous les spécialistes en médecine de famille le sont aussi en MIG.

\section{Epilogue}

Le développement d'un PFP commun s'était arrêté à mi-chemin en 2010 et le PFP en MIG reste loin derrière le modèle proposé par les internistes généralistes genevois Humier et Stalder (tronc commun) dans les années 90 [8]. Il marginalise la discipline de la médecine de famille, et ce au détriment de toutes les autres: de la MIG, qui perd un solide pilier de la médecine non interne; des soins de base ambulatoires, privés de la compétence et de l'expérience interdisciplinaires essentielles à la collaboration interprofessionnelle; de la médecine, qui se prive de la spécialité interdisciplinaire en étroite relation avec les sciences sociales et solidement implantée à l'interface entre médecine et société.

Une amélioration de l'actuel PFP serait facile et pourrait enfin déclencher l'élan tant espéré depuis 2010 pour la médecine de premier recours comme pour la médecine générale, qu'elles soient stationnaires ou ambulatoires.

\section{Crédit photo}

(c) Nikolai Lenets | Dreamstime.com

\section{Références}

1 Zeller A, Tschudi P. «Anamnese und Status» bei Schweizer Hausärzten. PrimaryCare. 2016;16(15):277-80. https://primary-hospital-care.ch/de/article/doi/phc-d.2016.01327/. 2 Gisler LB, Bachofner M, Moser-Bucher CN, Scherz N, Streit S. From practice employee to (co-)owner: young GPs predict their future careers: a cross-sectional survey. BMC Fam Pract. 2017;18(1):12. doi: 10.1186/s12875-017-0591-7.

3 Djalali S, Fre, A, Rosemann T, Tandjung R. [Vocational training for general practitioners - comparing Switzerland with otherEuropean countries]. Praxis. 2013;102:327-33. doi: 10.1024/1661-8157/a001225.

4 Bürgi U, Tschudi P, Briner V,Héritier F. Das neue Weiterbildungsprogramm Allgemeine Innere Medizin. PrimaryCare. 2010;10: 285-7. https://primary-hospital-care.ch/de/article/doi/pc-d.2010.08696/

5 FMH-Ärztestatistik 2017. Available from: https://www.fmh.ch/ services/statistik/aerztestatistik.html

6 Zahlen von der Befragung durch WB-Kommission SGAM/SGAIM an der FA-Prüfung (nicht publiziert).

7 Hausarzt oder Hausärztin: Beruf oder Berufung? Echo der ZeitRadio SRF, Minute 4:34.

8 Humier JP, Stalder S. Pérspectives pour la formation postgraduée de premier recours. http://www.hausarztmedizin.org/fileserver/ hs.pdf. 